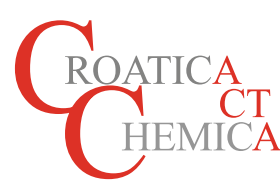

\title{
Visible Light Promoted Allylic C-H Oxidation
}

\author{
Vishal Srivastava, ${ }^{1}$ Pravin K. Singh, ${ }^{2}$ Praveen P. Singh ${ }^{3, *}$
}

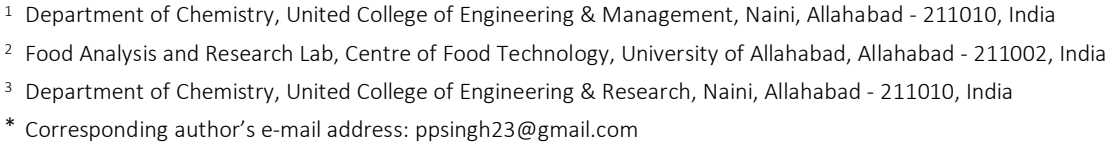

RECEIVED: March 15, 2017 * REVISED: July 15, 2017 * ACCEPTED: July 19, 2017

Abstract: A mild and efficient one-pot visible light-induced method has been developed for the allylic oxidation. The oxidation of allylic systems has played a prominent role in this context as possibly the most widely applied $\mathrm{C}-\mathrm{H}$ functionalization, owing to the utility of enones. The allylic $\mathrm{C}-\mathrm{H}$ oxidation is relatively simple and predictable, even on a preparative scale, because active species generated at the allylic position are stabilized by the double bond.

Keywords: eosin Y, visible-light, organophotoredox, allylic oxidation, C-H oxidation, enones.

\section{INTRODUCTION}

0 XIDATION plays an important role in various organic transformations because it not only adjusts the oxidation state of functional groups, but also introduces oxygen atoms to the carbon framework and allows further functional group transformations. The direct functionalization of $\mathrm{C}-\mathrm{H}$ bonds are beginning to reshape the field of retrosynthetic analysis, affecting the synthesis of natural products, medicines and materials. ${ }^{[1]}$ The oxidation of alIylic systems has played a prominent role in this context as possibly the most widely applied $\mathrm{C}-\mathrm{H}$ functionalization, owing to the utility of enones and allylic alcohols as versatile intermediates, and their prevalence in natural and unnatural materials. ${ }^{[2]}$ Various oxidative conversions are used extensively in synthetic organic chemistry. ${ }^{[3,4]}$ An oxidation reaction of special interest is the allylic oxidation that allows the conversion of alkenes into allyl alcohols or enones. ${ }^{[5]}$ Allylic oxidations via the direct $\mathrm{sp}^{3} \mathrm{C}-\mathrm{H}$ bond activation of alkenes and alkylarenes to the corresponding $\alpha, \beta$ - unsaturated enones and carbonyl compounds are important transformations with a wide variety of industrial applications ${ }^{[6,7]}$ including the synthesis of drug precursors and building blocks in many organic syntheses.

The potential of developing new synthetic methodologies using visible light has recently received much attention from a number of research groups..$^{[8]}$ This is because solar energy (visible light) is clean, easy to handle and an unlimited energy source having great prospects for the development of sustainable and eco-friendly protocols for organic synthesis. ${ }^{[9]}$ Some pioneering researchers have dedicated to converting solar energy into chemical energy for chemical transformations ${ }^{[10,11]}$ which includes a promising strategy for the application of photoredox catalysts to initiate single electron transfer processes have been developed. ${ }^{[12,13]}$ The visible light photoredox catalysis has recently received much attention in organic synthesis owing to readily availability, sustainability, non-toxicity and ease of handling of visible light. ${ }^{[14-19]}$

Recently, a superior alternative to transition metal photoredox catalysts, especially metal-free organic dyes such as eosin $Y$, fluorescein, rose bengal, nile red, perylene and rhodamine $B$ have been used as economically and ecologically superior surrogates for Ru(II) and Ir(II) complexes in visible-light promoted organic transformations involving $\mathrm{SET}^{[20-23]}$ (single electron transfer). These organic dyes have got much more attention with the last few years also due to easy handling, eco-friendly and have great potential for applications in visible-light-mediated organic synthesis[2427] which fulfils the basic principle of green chemistry. Encouraged by organocatalytic visible light- mediated aerobic oxidative transformations ${ }^{[28,29]}$ and in continuation of our 
work on development of novel environmentally benign synthesis ${ }^{[30-35]}$ herein we report a simple, visible light irradiated, efficient and green protocol for the synthesis of substituted cyclohexenones via allylic $\mathrm{C}-\mathrm{H}$ oxidation. Cyclohexenones have great utility as it serves as potential bioactive compounds and exhibit a variety of pharmacological properties like anticancer, antitumour, antiasthmetic and antipyretic. ${ }^{[36-40]}$

\section{EXPERIMENTAL}

Melting points were determined by an open glass capillary method and are uncorrected. All chemicals used were reagent grade and were used as received. ${ }^{1} \mathrm{H}$ NMR and ${ }^{13} \mathrm{C}$ NMR spectra were recorded on a Bruker AVANCE DPX ( $400 \mathrm{MHz}$ and $75 \mathrm{MHz}$ ) FT spectrometer in DMSO using TMS as an internal reference (chemical shift in $\delta, \mathrm{ppm}$ ). Mass spectra were recorded on JEOL SX-303 (FAB) mass spectrophotometer. Elemental analyses were carried out using a Coleman automatic $\mathrm{C}, \mathrm{H}, \mathrm{N}$ analyser.

\section{General Procedure for the Photocatalysed Synthesis of Substituted Cyclohexenone}

A round bottom flask was charged with cyclohexene 1(a-l) (1.0 mmol), eosin $\mathrm{Y}(2 \mathrm{~mol} \%)$ and $\mathrm{CH}_{3} \mathrm{CN}(3 \mathrm{~mL})$ and the contents were stirred in open air under irradiation with
Luxeon Rebel high power green LEDs [2.50 W, $\lambda=535 \mathrm{~nm}$ ] at room temperature for $40-180 \mathrm{~min}$. After the completion of reaction (as indicated by TLC), it was quenched with water $(5 \mathrm{~mL})$ and extracted with ethyl acetate $(3 \times 5 \mathrm{~mL})$. The organic phase was dried over anhydrous magnesium sulfate and concentrated under reduced pressure to yield the crude product, which was purified by silica gel column chromatography using a mixture of EtOAc-Hexane to give the pure product $2(a-1)$ in high yields (76-97\%).

2',5'-difluoro-5,6-dihydro-[1,1'-biphenyl]-3(4H)-one (2a): m.p. $72{ }^{\circ} \mathrm{C}, \mathrm{m} / \mathrm{z}$ : 208.07; Mol. Wt: 208.21. ${ }^{1 \mathrm{H}} \mathrm{NMR}(400 \mathrm{MHz}$, $\left.\mathrm{CDCl}_{3}\right) \delta: 7.13-6.94(\mathrm{~m}, 3 \mathrm{H}), 6.27(\mathrm{~d}, 1 \mathrm{H}, J=1.7 \mathrm{~Hz}), 2.72(\mathrm{tt}$, $2 \mathrm{H} J=6.1,1.7 \mathrm{~Hz}), 2.51-2.16(\mathrm{~m}, 4 \mathrm{H}) ;{ }^{13} \mathrm{CNMR}(101 \mathrm{MHz}$, $\mathrm{CDCl}_{3}$ ) $\delta: 199.1,158.6155 .8,155.4,129.4$ 129.0, 117.5, 117.1, 115.2, 37.3, 29.4, 29.3, 23.0. Anal. Calcd. for $\mathrm{C}_{12} \mathrm{H}_{10} \mathrm{~F}_{2} \mathrm{O}: \mathrm{C}$, 69.23: $H, 4.84: F, 18.25$. Found: $C, 69.20: H, 4.81 ; F, 18.20$.

2',5'-dichloro-5,6-dihydro-[1,1'-biphenyl]-3(4H)-one (2b): m.p. $96{ }^{\circ} \mathrm{C}, \mathrm{m} / \mathrm{z}$ : 240.01; Mol. Wt: 241. 11; ${ }^{1} \mathrm{H}$ NMR (400 $\left.\mathrm{MHz}, \mathrm{CDCl}_{3}\right):{ }^{1} \mathrm{H} \mathrm{NMR}\left(400 \mathrm{MHz}, \mathrm{CDCl}_{3}\right) \delta: 7.31-7.23(\mathrm{~m}$, $3 \mathrm{H}), 6.04(\mathrm{~s}, 1 \mathrm{H}), 2.65(\mathrm{td}, 2 \mathrm{H}, J=6.0,1.6 \mathrm{~Hz}), 2.51(\mathrm{dd}, 2 \mathrm{H}$, $J=7.4,6.0 \mathrm{~Hz}), 1.94-3.16(\mathrm{~m}, 2 \mathrm{H}) ;{ }^{13} \mathrm{C}$ NMR $(101 \mathrm{MHz}$, $\left.\mathrm{CDCl}_{3}\right)$ \&: 198.9, 159.4, 141.0, 132.9, 131.1, 129.70, 129.6, 129.5, 128.7, 37.3, 30.1, 23.0. Anal. Calcd. for $\mathrm{C}_{12} \mathrm{H}_{10} \mathrm{Cl}_{2} \mathrm{O}: \mathrm{C}$, 59.78: $\mathrm{H}, 4.18: \mathrm{Cl}, 29.41$. Found: $\mathrm{C}, 59.72: \mathrm{H}, 4.16 ; \mathrm{Cl}, 29.38$.

Table 1.
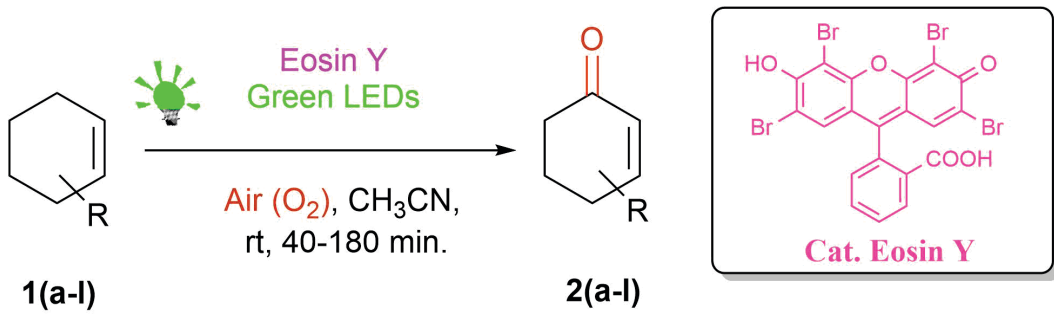

\begin{tabular}{|c|c|c|c|c|}
\hline Entry & $\mathrm{R}$ & Product & Time (min) & Yield (\%) \\
\hline 1 & $2,5-\mathrm{F} \cdot \mathrm{C}_{6} \mathrm{H}_{3}$ & $2 a$ & 140 & 76 \\
\hline 2 & $2,5-\mathrm{Cl} \cdot \mathrm{C}_{6} \mathrm{H}_{3}$ & $2 b$ & 140 & 79 \\
\hline 3 & $2-\mathrm{Cl} .3-\mathrm{CH}_{3} \cdot \mathrm{C}_{6} \mathrm{H}_{3}$ & $2 c$ & 100 & 84 \\
\hline 4 & $2-\mathrm{CH}_{3} \cdot 3-\mathrm{Cl} \cdot \mathrm{C}_{6} \mathrm{H}_{3}$ & $2 d$ & 40 & 84 \\
\hline 5 & 2-F. $\mathrm{C}_{6} \mathrm{H}_{4}$ & $2 \mathrm{e}$ & 80 & 78 \\
\hline 6 & 4-F. $\mathrm{C}_{6} \mathrm{H}_{4}$ & $2 f$ & 140 & 77 \\
\hline 7 & $2-\mathrm{OCH}_{3} \cdot \mathrm{C}_{6} \mathrm{H}_{4}$ & $2 \mathrm{~g}$ & 140 & 93 \\
\hline 8 & $3-\mathrm{OCH}_{3} \cdot \mathrm{C}_{6} \mathrm{H}_{4}$ & $2 \mathrm{~h}$ & 180 & 90 \\
\hline 9 & $2,5-\mathrm{OCH}_{3} \cdot \mathrm{C}_{6} \mathrm{H}_{3}$ & $2 \mathrm{i}$ & 180 & 97 \\
\hline 10 & $2,5-\mathrm{CH}_{3} \cdot \mathrm{C}_{6} \mathrm{H}_{3}$ & $2 \mathrm{j}$ & 40 & 80 \\
\hline 11 & $3-\mathrm{C}\left(\mathrm{CH}_{3}\right)_{3} \cdot \mathrm{C}_{6} \mathrm{H}_{4}$ & $2 k$ & 40 & 79 \\
\hline 12 & 4- $\mathrm{C}\left(\mathrm{CH}_{3}\right)_{3} \cdot \mathrm{C}_{6} \mathrm{H}_{4}$ & 21 & 40 & 78 \\
\hline
\end{tabular}


2'-chloro-3'-methyl-5,6-dihydro-[1,1'-biphenyl]-3(4H)-one (2c): m.p. $88^{\circ} \mathrm{C}, \mathrm{m} / \mathrm{z}$ : 220.07; Mol. Wt: $220.70^{1} \mathrm{H}$ NMR $(400 \mathrm{MHz}$, $\left.\mathrm{CDCl}_{3}\right) \delta: 7.22(\mathrm{dd}, 1 \mathrm{H}, J=1.8,0.8 \mathrm{~Hz}), 7.17(\mathrm{t}, 1 \mathrm{H}, J=7.5$ $\mathrm{Hz}$ ), 7.00 (dd, $1 \mathrm{H}, J=7.4,1.8 \mathrm{~Hz}), 6.01(\mathrm{~s}, 1 \mathrm{H}), 2.66-2.51(\mathrm{~m}$, 4H), $2.41(\mathrm{~s}, 3 \mathrm{H}), 2.17$ (dd, $2 \mathrm{H}, J=8.0,6.2 \mathrm{~Hz}) ;{ }^{13} \mathrm{CNMR}(101$ $\left.\mathrm{MHz}, \mathrm{CDCl}_{3}\right) \delta: 199.6,162.2,140.3,137.2,131.3,130.9$, 129.0, 126.5, 126.2, 37.4, 30.6, 23.1, 20.5. Anal. Calcd. for $\mathrm{C}_{13} \mathrm{H}_{13} \mathrm{ClO}: \mathrm{C}, 70.75: \mathrm{H}, 5.94: \mathrm{Cl}, 16.06$. Found: $\mathrm{C}, 70.72$ : $\mathrm{H}, 5.92 ; \mathrm{Cl}, 16.02$.

3'-chloro-2'-methyl-5,6-dihydro-[1,1'-biphenyl]-3(4H)-one (2d): m.p. $110^{\circ} \mathrm{C}, \mathrm{m} / \mathrm{z}$ : 220.07; Mol. Wt: 220.70; ${ }^{1} \mathrm{H}$ NMR (400 $\left.\mathrm{MHz}, \mathrm{CDCl}_{3}\right) \delta: 7.35(\mathrm{dd}, 1 \mathrm{H}, J=8.0,1.3 \mathrm{~Hz}), 7.14-7.48(\mathrm{~m}$, $1 \mathrm{H}), 6.99(\mathrm{dd}, 1 \mathrm{H}, J=7.7,1.3 \mathrm{~Hz}), 5.96(\mathrm{~s}, 1 \mathrm{H}), 2.54-2.17(\mathrm{~m}$, 6H), $2.31(\mathrm{~s}, 3 \mathrm{H}) ;{ }^{13} \mathrm{CNMR}\left(101 \mathrm{MHz}, \mathrm{CDCl}_{3}\right) \delta: 199.2,162.8$, 142.7, 135.5, 132.2, 129.1, 129.0, 126.9, 125.3, 37.3, 31.4, 23.1, 17.5. Anal. Calcd. for $\mathrm{C}_{13} \mathrm{H}_{13} \mathrm{ClO}: \mathrm{C}, 70.75: \mathrm{H}, 5.94: \mathrm{Cl}$, 16.06. Found: $\mathrm{C}, 70.72: \mathrm{H}, 5.92 ; \mathrm{Cl}, 16.02$.

2'-fluoro-5,6-dihydro-[1,1'-biphenyl]-3(4H)-one (2e): m.p. $78{ }^{\circ} \mathrm{C}, \mathrm{m} / \mathrm{z}$ : 190.08; Mol. Wt: 190.22 ; ${ }^{1} \mathrm{H}$ NMR (400 $\left.\mathrm{MHz}, \mathrm{CDCl}_{3}\right) \delta: 7.34-7.14(\mathrm{~m}, 2 \mathrm{H}), 7.17(\mathrm{td}, 1 \mathrm{H}, J=7.7,1.2$ $\mathrm{Hz}), 7.10(\mathrm{dd}, 1 \mathrm{H}, J=11.3,8.1,1.2 \mathrm{~Hz}), 6.28(\mathrm{~d}, 1 \mathrm{H}, J=1.7$ $\mathrm{Hz}), 2.76(\mathrm{tt}, 2 \mathrm{H}, J=6.1,1.7 \mathrm{~Hz}), 2.50(\mathrm{dd}, 2 \mathrm{H}, J=7.6,5.8$ $\mathrm{Hz}), 3.16-1.94(\mathrm{~m}, 2 \mathrm{H}) ;{ }^{13} \mathrm{C} \mathrm{NMR}\left(101 \mathrm{MHz}, \mathrm{CDCl}_{3}\right) \delta: 199.4$, $159.83,157.1,130.9,128.9,127.8,124.4,116.4,37.4,29.7$, 29.6, 23.1. Anal. Calcd. for $\mathrm{C}_{12} \mathrm{H}_{11}$ FO: C, 75.77: H, 5.83: F, 9.99. Found: $C, 75.72: H, 5.79 ; F, 9.95$.

\section{4'-fluoro-5,6-dihydro-[1,1'-biphenyl]-3(4H)-one (2f):} m.p. $130{ }^{\circ} \mathrm{C}, \mathrm{m} / \mathrm{z}$ : 190.08.; Mol. Wt: $190.22 ;{ }^{1} \mathrm{H}$ NMR (400 $\left.\mathrm{MHz}, \mathrm{CDCl}_{3}\right) \delta: 7.53(\mathrm{dd}, 2 \mathrm{H}, J=8.9,5.2 \mathrm{~Hz}), 7.10(\mathrm{t}, 2 \mathrm{H}, J$ $=8.6 \mathrm{~Hz}), 6.37(\mathrm{~s}, 1 \mathrm{H}), 2.75(\mathrm{td}, 2 \mathrm{H}, J=6.0,1.5 \mathrm{~Hz}), 3.16-$ $1.94(\mathrm{~m}, 2 \mathrm{H}), 2.16(\mathrm{dd}, 2 \mathrm{H}, J=8.6,4.8 \mathrm{~Hz}) ;{ }^{13} \mathrm{C}$ NMR (101 $\left.\mathrm{MHz}, \mathrm{CDCl}_{3}\right) \delta:$ 199.6, 163.8, 158.4, 134.9, 130.3, 128.0, 126.2, 125.3, 115.81, 37.1, 28.2, 22.7. Anal. Calcd. for $\mathrm{C}_{12} \mathrm{H}_{11}$ FO: C, 75.77: H, 5.83: F, 9.99. Found: C, 75.72: $\mathrm{H}, 5.79 ; \mathrm{F}, 9.95$.

\section{3-(2-methoxyphenyl)cyclohex-2-enone (2g):}

m.p. $126{ }^{\circ} \mathrm{C}, \mathrm{m} / \mathrm{z}$ : 202.10; Mol. Wt: 202.25; ${ }^{1} \mathrm{H}$ NMR (400 $\mathrm{MHz}, \mathrm{CDCl}_{3}$ ) $\delta: 7.27(\mathrm{dd}, 1 \mathrm{H}, J=8.3,1.8 \mathrm{~Hz}), 7.13(\mathrm{dd}, 1 \mathrm{H}, J$ $=7.6,1.8 \mathrm{~Hz}), 7.34-7.02(\mathrm{~m}, 2 \mathrm{H}), 6.13(\mathrm{~s}, 1 \mathrm{H}), 3.77(\mathrm{~s}, 3 \mathrm{H})$, $2.67(\mathrm{td}, \mathrm{H}, J=6.0,1.6 \mathrm{~Hz}), 2.41(\mathrm{dd}, 2 \mathrm{H}, J=7.4,6.0 \mathrm{~Hz})$, 3.16-1.94 (m, 2H); ${ }^{13} \mathrm{C}$ NMR (101 MHz, $\left.\mathrm{CDCl}_{3}\right) \delta: 200.0$, 161.6, 156.6, 130.2, 129.7, 128.7,128.2, 120.7, 111.2, 55.4, 37.5, 30., 23.3. Anal. Calcd. for $\mathrm{C}_{13} \mathrm{H}_{14} \mathrm{O}_{2}: \mathrm{C}, 77.20: \mathrm{H}, 6.98$. Found: C, 77.17: $\mathrm{H}, 6.95$.

\section{3-(3-methoxyphenyl)cyclohex-2-enone (2h):}

m.p. $140{ }^{\circ} \mathrm{C}, \mathrm{m} / \mathrm{z}$ : 202.10; Mol. Wt: $202.25 ;{ }^{1} \mathrm{H}$ NMR (400 $\left.\mathrm{MHz}, \mathrm{CDCl}_{3}\right) \delta: 7.32(\mathrm{t}, 1 \mathrm{H}, J=8.0 \mathrm{~Hz}), 7.48-6.51(\mathrm{~m}, 2 \mathrm{H})$, $6.95(\mathrm{dd}, 1 \mathrm{H}, J=8.2,0.9 \mathrm{~Hz}), 6.41(\mathrm{~s}, 1 \mathrm{H}), 3.84(\mathrm{~s}, 3 \mathrm{H}), 2.76$ $(\mathrm{td}, 2 \mathrm{H}, J=6.0,1.6 \mathrm{~Hz}), 3.16-1.94(\mathrm{~m}, 2 \mathrm{H}), 2.16(\mathrm{dd}, 2 \mathrm{H}$, $J=7.1,1.1 \mathrm{~Hz}) ;{ }^{13} \mathrm{C} \mathrm{NMR}\left(101 \mathrm{MHz}, \mathrm{CDCl}_{3}\right) \delta: 199.9,159.8$,
159.7, 140.3, 129.7, 125.7, 118.6, 115.5, 111.8, 55.4, 37.3, 28.2, 22.8. Anal. Calcd. for $\mathrm{C}_{13} \mathrm{H}_{14} \mathrm{O}_{2}$ : C, 77. 20: H, 6.98 . Found: C, 77.17: H,6.95.

2',5'-dimethoxy-5,6-dihydro-[1,1'-biphenyl]-3(4H)-one (2i): m.p. $137{ }^{\circ} \mathrm{C}, \mathrm{m} / \mathrm{z}$ : 232.11; Mol. Wt: 232.28; ${ }^{1} \mathrm{H}$ NMR (400 $\left.\mathrm{MHz}, \mathrm{CDCl}_{3}\right) \delta: 6.89-6.73(\mathrm{~m}, 3 \mathrm{H}), 6.20(\mathrm{~s}, 1 \mathrm{H}), 3.78(\mathrm{~d}, 6 \mathrm{H}$, $J=5.1 \mathrm{~Hz}), 2.73-2.12(\mathrm{~m}, 4 \mathrm{H}), 2.48(\mathrm{dd}, 2 \mathrm{H}, J=7.4,6.0 \mathrm{~Hz}$ ); ${ }^{13} \mathrm{C}$ NMR $\left(101 \mathrm{MHz}, \mathrm{CDCl}_{3}\right) \delta: 200.0,161.4,153.6,150.8$, 130.5, 128.3, 115.0, 114.5, 112.5, 56.1, 55.8, 37.5, 30.0, 23.2. Anal. Calcd. for $\mathrm{C}_{14} \mathrm{H}_{16} \mathrm{O}_{3}: \mathrm{C}, 72$. 39: $\mathrm{H}, 6.94$. Found: $\mathrm{C}$, 72.36: H,6.90.

2',5'-dimethyl-5,6-dihydro-[1,1'-biphenyl]-3(4H)-one (2j): m.p. $90{ }^{\circ} \mathrm{C}, \mathrm{m} / \mathrm{z}$ : 200.12; Mol. Wt: 200.28; ${ }^{1 \mathrm{H}}$ NMR (400 $\left.\mathrm{MHz}, \mathrm{CDCl}_{3}\right) \delta: 7.11-6.89(\mathrm{~m}, 2 \mathrm{H}), 6.92(\mathrm{~d}, 1 \mathrm{H}, J=1.8 \mathrm{~Hz})$, $5.95(\mathrm{~s}, 1 \mathrm{H}), 2.60(\mathrm{td}, 2 \mathrm{H}, J=6.0,1.6 \mathrm{~Hz}), 2.47(\mathrm{dd}, 2 \mathrm{H}, J=$ 7.4, $6.0 \mathrm{~Hz}$ ), $2.33(\mathrm{~s}, 3 \mathrm{H}), 2.24(\mathrm{~s}, 3 \mathrm{H}), 2.14(\mathrm{dd}, 2 \mathrm{H}, J=7.0$, $5.9 \mathrm{~Hz}) ;{ }^{13} \mathrm{C}$ NMR $\left(101 \mathrm{MHz}, \mathrm{CDCl}_{3}\right) \delta: 199.6,163.7,138.2$, $138.2,137.8,133.8,131.4,128.6,126.9,126.5,123.9,37.3$, 31.3, 23.1, 21.0, 19.9. Anal. Calcd. for $\mathrm{C}_{14} \mathrm{H}_{16} \mathrm{O}: \mathrm{C}, 83.96: \mathrm{H}$, 8.05. Found: $\mathrm{C}, 83.92: \mathrm{H}, 8.03$.

3'-(tert-butyl)-5,6-dihydro-[1,1'-biphenyl]-3(4H)-one (2k): m.p. $112{ }^{\circ} \mathrm{C}, \mathrm{m} / \mathrm{z}$ : 228.15; Mol. Wt: 228.34; ${ }^{1 \mathrm{H}} \mathrm{NMR}(400$ $\left.\mathrm{MHz}, \mathrm{CDCl}_{3}\right) \delta: 7.56(\mathrm{~s}, 1 \mathrm{H}), 7.45(\mathrm{dd}, 1 \mathrm{H}, J=5.2,2.0 \mathrm{~Hz}$ ), $7.35-6.63(\mathrm{~m}, 2 \mathrm{H}), 6.43(\mathrm{~s}, 1 \mathrm{H}), 2.79(\mathrm{td}, 2 \mathrm{H}, J=6.1,1.5 \mathrm{~Hz})$, 2.49 (dd, $2 \mathrm{H}, J=7.4,6.0 \mathrm{~Hz}), 2.16(\mathrm{dd}, 2 \mathrm{H}, J=7.8,5.9 \mathrm{~Hz}$ ), 1.35 (s, 9H); ${ }^{13} \mathrm{C}$ NMR (101 MHz, $\left.\mathrm{CDCl}_{3}\right) \delta: 199.9,160.5$, $151.7,138.6,128.5,127.1,125.4,123.3,123.0,37.3,34.8$, 31.3, 28.3, 22.9. Anal. Calcd. for $\mathrm{C}_{16} \mathrm{H}_{20} \mathrm{O}: \mathrm{C}, 84.16: \mathrm{H}, 8.83$. Found: C, 84.12: $\mathrm{H}, 8.80$.

4'-(tert-butyl)-5,6-dihydro-[1,1'-biphenyl]-3(4H)-one (2I): m.p. $155^{\circ} \mathrm{C}, \mathrm{m} / \mathrm{z}$ : 228.15; Mol. Wt: 228.34; ${ }^{1} \mathrm{H}$ NMR (400 $\left.\mathrm{MHz}, \mathrm{CDCl}_{3}\right) \delta: 7.50(\mathrm{~d}, 2 \mathrm{H}, J=8.8 \mathrm{~Hz}), 7.43(\mathrm{~d}, 2 \mathrm{H}, J=8.8$ $\mathrm{Hz}), 6.43(\mathrm{~s}, 1 \mathrm{H}), 2.77(\mathrm{td}, 2 \mathrm{H}, J=6.1,1.6 \mathrm{~Hz}), 2.48(\mathrm{dd}, 2 \mathrm{H}$, $J=7.4,5.9 \mathrm{~Hz}), 3.16-1.94(\mathrm{~m}, 2 \mathrm{H}), 1.34(\mathrm{~s}, 9 \mathrm{H}) ;{ }^{13} \mathrm{C} \mathrm{NMR}$ (101 MHz, CDCl3) $\delta: 199.9,159.5,153.5,135.7,125.9$, 125.7, 124.7, 37.3, 34.8,31.2, 28.0, 22.8. Anal. Calcd. for $\mathrm{C}_{16} \mathrm{H}_{20} \mathrm{O}: \mathrm{C}, 84.16: \mathrm{H}, 8.83$. Found: C, 84.12: $\mathrm{H}, 8.80$.

\section{RESULTS AND DISCUSSION}

In order to realise our idea and optimise the reaction conditions, the key reaction of cyclohexene 1a with catalytic amount of eosin $Y$ in a solvent under irradiation with green LEDs [2.50 W, $\lambda=535 \mathrm{~nm}$ ] in open air (Table 2). We were delighted to get the desired product substituted cyclohexenone $\mathbf{2 a}$ in $92 \%$ yield (Table 2, entry 1 ). Then, the control experiments were carried out, which show that eosin $\mathrm{Y}$, air $\left(\mathrm{O}_{2}\right)$ and visible light are essential for the reaction, because in the absence of any of the reagents/reaction parameters either the product was not detected (n. d.) or formed in the traces (Table 2, entry 1 versus $12-15$ ). The optimum 
Table 2. Optimisation of reaction conditions. ${ }^{(a)}$

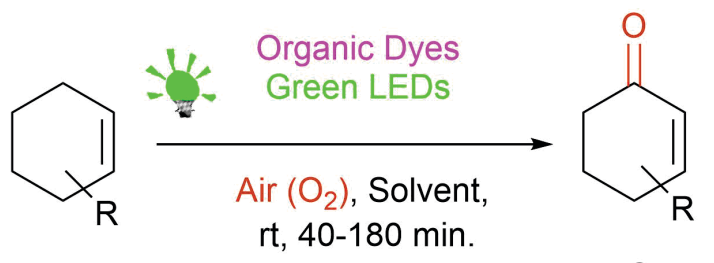

$1 a$

$2 a$

\begin{tabular}{|c|c|c|c|c|}
\hline Entry & Catalyst (mol\%) & Solvent & Time (min) & Yield $(\%)^{(b)}$ \\
\hline 1 & eosin $Y(2)$ & $\mathrm{CH}_{3} \mathrm{CN}$ & 40 & 92 \\
\hline 2 & rose bengal (2) & $\mathrm{CH}_{3} \mathrm{CN}$ & 40 & 72 \\
\hline 3 & fluorescein (2) & $\mathrm{CH}_{3} \mathrm{CN}$ & 40 & 70 \\
\hline 4 & nile red (2) & $\mathrm{CH}_{3} \mathrm{CN}$ & 40 & 74 \\
\hline 5 & perylene (2) & $\mathrm{CH}_{3} \mathrm{CN}$ & 40 & 75 \\
\hline 6 & rhodamine B (2) & $\mathrm{CH}_{3} \mathrm{CN}$ & 40 & 76 \\
\hline 7 & $\operatorname{eosin} Y(2)$ & $\mathrm{EtOH}$ & 40 & 63 \\
\hline 8 & $\operatorname{eosin} Y(2)$ & DMF & 40 & 78 \\
\hline 9 & $\operatorname{eosin} Y(2)$ & DMSO & 40 & 86 \\
\hline 10 & $\operatorname{eosin} Y(1)$ & $\mathrm{CH}_{3} \mathrm{CN}$ & 40 & 67 \\
\hline 11 & $\operatorname{eosin} Y(3)$ & $\mathrm{CH}_{3} \mathrm{CN}$ & 40 & 92 \\
\hline 12 & $\operatorname{eosin} Y(2)$ & $\mathrm{CH}_{3} \mathrm{CN}$ & 180 & traces $^{(c)}$ \\
\hline 13 & $\operatorname{eosin} Y(2)$ & $\mathrm{CH}_{3} \mathrm{CN}$ & 180 & traces $^{(d)}$ \\
\hline 14 & - & $\mathrm{CH}_{3} \mathrm{CN}$ & 180 & n. d. (e) \\
\hline 15 & $\operatorname{eosin} Y(2)$ & $\mathrm{CH}_{3} \mathrm{CN}$ & 180 & traces $^{(f)}$ \\
\hline 16 & $\operatorname{eosin} Y(2)$ & $\mathrm{CH}_{3} \mathrm{CN}$ & 180 & $58^{(g)}$ \\
\hline 18 & $\operatorname{eosin} Y(2)$ & $\mathrm{CH}_{3} \mathrm{CN}$ & 40 & $92^{(\mathrm{h})}$ \\
\hline 19 & $\operatorname{eosin} Y(2)$ & $\mathrm{CH}_{3} \mathrm{CN}$ & 40 & $89^{(\mathrm{i})}$ \\
\hline
\end{tabular}

(a) Reaction conditions: 1a (1.0 mmol), catalyst (mol\%), in $3 \mathrm{~mL}$ solvent irradiated using Luxeon Rebel high power green LEDs [2.50 W, $\lambda=535 \mathrm{~nm}]$ under an air atmosphere at rt for $40-180 \mathrm{~min}$.

(b) Another one Isolated yield of the pure product $2 a$.

(c) Reaction was performed under nitrogen.

(d) Reaction was performed in the dark.

(e) Reaction was carried out without catalyst.

Reaction was quenched with 2,2,6,6-tetramethylpiperidyl-1-oxyl (TEMPO) (1.0 mmol).

(g) 18 W CFL (compact fluorescent lamp, Philips) was used.

(h) $\mathrm{O}_{2}$ balloon was used.

(i) The reaction was quenched with DABCO $(1.0 \mathrm{mmol})$.

amount of eosin $Y$ required for the reaction was $2 \mathrm{~mol} \%$. On decreasing the amount of eosin $Y$ from $2 \mathrm{~mol} \%$ to $1 \mathrm{~mol} \%$ the yield was considerably reduced (Table 2, entry 10), whereas the yield was not enhanced even on use of $3 \mathrm{~mol} \%$ of eosin Y (Table 2, entry 11). The use of another organic photocatalyst like rose bengal (2 mol\%), fluorescein ( 2 mol\%), nile red ( 2 mol\%), perylene $(2 \mathrm{~mol} \%)$ and rhodamine $\mathrm{B}$ ( 2 mol\%) was not so effective as eosin Y (2 mol\%) (Table 2, entry 1 versus 2-6).

Green LEDs [2.50 W, $\lambda=535 \mathrm{~nm}$ ] were more effective than fluorescent light (Table 2, entry 16), which shows that the higher photocatalytic activity of eosin $\mathrm{Y}$ in presence of high intensity green light. The presence of $\mathrm{O}_{2}$ (air) is also essential for the reaction because only a trace of product formation was detected under a nitrogen atmosphere (Table 2, entry 12). Notably, the same result was obtained on using $\mathrm{O}_{2}$ (balloon) instead of an air atmosphere (Table 2, entry 18). Moreover, the reaction was quenched with 2,2,6,6-tetramethylpiperidyl-1-oxyl (TEMPO) $(1.0 \mathrm{mmol}$ ) in standard state showing that there may be radical intermediates involved in reaction (Table 2, entry 15). Furthermore, the reaction did not quench in the presence of DABCO 


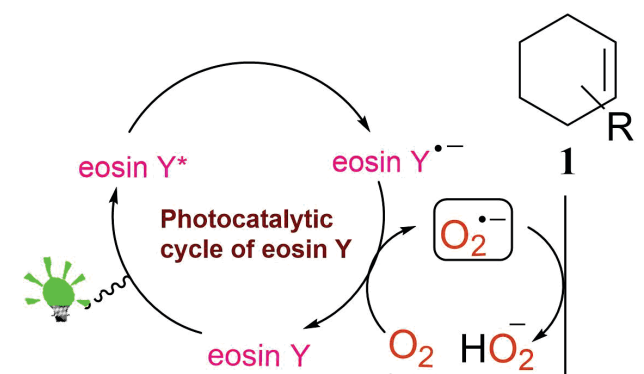<smiles>[R]C1=CC(O)CCC1=O</smiles>

2<smiles>[R]C1C=CC(OO[O-])CCC1</smiles>

B
.

(air)

\section{$\mathrm{O}_{2}$ (air)}<smiles>Cc1ccccc1CCc1ccccc1C(=O)O</smiles>

Eosin $Y$<smiles></smiles>

$\operatorname{Eosin} \mathbf{Y}^{*-}$

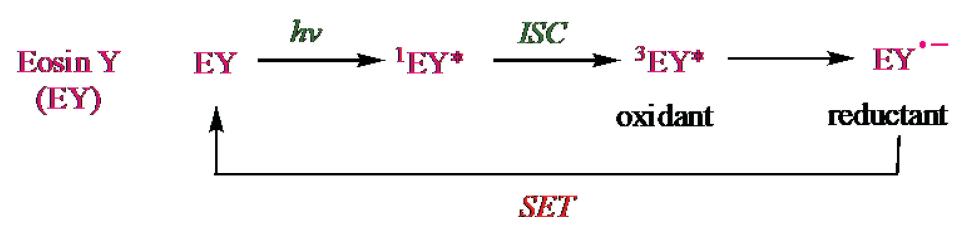

Photoredox catalytic cycle of eosin $Y$

Scheme 1. Plausible mechanism for the formation of substituted cyclohexanone.

(1.0 mmol) (Table 2, entry 19), which indicates the involvement of triplet oxygen in the reaction. Since, DABCO is a good quencher of singlet oxygen and quenching was not observed, it suggests that singlet oxygen was not involved in the reaction. ${ }^{[41]}$ The possibility of cyclohexne as a reducing agent that undergoes oxidation to radical cation is very less because quenching of radical cation could not be done. Next, the reaction was optimized for an effective solvent system. It was found that $\mathrm{CH}_{3} \mathrm{CN}$ was the best among the tested solvents $\mathrm{EtOH}, \mathrm{DMF}, \mathrm{DMSO}$ and $\mathrm{CH}_{3} \mathrm{CN}$, hence it was used throughout the present study (Table 2, entry 1 versus 7-9). The scope of the present protocol across a range of cyclohexene incorporating various substituents. It was found that cyclohexene with an electron-donating group on the aromatic ring appear to react faster and afford marginally higher yields in compar ison to those bearing an electron-withdrawing group.

On the basis of our observations and the literature reports, ${ }^{[42,43]}$ a plausible mechanistic pathway is depicted in Scheme 1. On absorption of visible light, the organophotoredox catalyst eosin $\mathrm{Y}(\mathrm{EY})$ is excited to its singlet state ${ }^{1} \mathrm{EY} *$ which through inter system crossing (ISC) comes to its more stable triplet state ${ }^{3} \mathrm{EY}^{*}$ and undergoes a single electron transfer (SET). ${ }^{3} \mathrm{EY}^{*}$ may undergo both reductive and oxidative quenching. ${ }^{[44-48]}$

A SET from substituted cyclohexene 1 generates allyl free radical $A$, which combines with $\mathrm{O}_{2}$ to form a peroxy radical $B$, which is followed by elimination of $\mathrm{OH}^{\circ}$ to give the desired product 2 . The formation of superoxide radical anion $\left(\mathrm{O}_{2}^{--}\right)$during the reaction was confirmed by the detection of the resulting $\mathrm{H}_{2} \mathrm{O}_{2}$ using $\mathrm{Kl} /$ starch indicator. ${ }^{[49]}$ 


\section{CONCLUSION}

In conclusion, we have developed a novel, one-pot procedure for the synthesis of substituted cyclohexenone from substituted cyclohexene via photocatalysed allylic oxidation. The protocol utilizes atmospheric oxygen and visible light as the cheapest and eco sustainable reagents and eo$\sin \mathrm{Y}$ as an organophotoredox catalyst at room temperature. Thus, it is a superior alternative to the existing method with respect to green and sustainable chemistry (better atomand step-economy) for the allylic oxidation reactions.

Acknowledgment. We sincerely thank Emerging Science Society for financial support as well as SAIF, CDRI, Lucknow and IISC Banglaore for providing microanalyses and spectra.

Supplementary Information. Supporting information to the paper is attached to the electronic version of the article at: http://doi.org/10.5562/cca3126.

\section{REFERENCES}

[1] W. R. Gutekunst, P. S. Baran, Chem. Soc. Rev. 2011, 40, 1976.

[2] V. Weidmann, W. Maison, Synthesis 2013, 45, 2201.

[3] K. B. Wilberg, Oxidation in Organic Chemistry; Academic Press: New York 1965.

[4] G. Cainelli, G. Cardille, Chromium in Oxidation Chemistry; Springer Verlag: Berlin 1984.

[5] P. C. B. Page, T. McCarthy, Comprehensive Organic Synthesis, Pergamon, Oxford, UK 1991, 7, 83.

[6] L. Kesavan, R. Tiruvalam, M. H. Ab Rahim, M. I. Bin Saiman, D. I. Enache, R. L. Jenkins, N. Dimitratos, J. A. Lopez-Sanchez, S. H. Taylor, D. W. Knight, C. J. Kiely, G. J. Hutchings, Science 2011, 331, 195.

[7] A. Aguadero, H. Falcon, J. M. Campos-Martin, S. M. Al-Zahrani, J. L. G. Fierro, J. A. Alonso, Angew. Chem. Int. Ed. 2011, 50, 6557.

[8] J. W. Tucker, C. R. J. Stephenson, J. Org. Chem. 2012, 77, 1617.

[9] X. Sala, I. Romero, M. Rodriguez, L. Escriche, A. Llobet, Angew. Chem. Int. Ed. 2009, 48, 2842.

[10] D. Mandler, I. Willner, J. Am. Chem. Soc. 1984, 106, 5352.

[11] O. Ishitani, S. Yanagida, S. Takamuku, C. Pac, J. Org. Chem. 1987, 52, 2790.

[12] A. Inagaki, M. Akita, Coord. Chem. Rev. 2010, 254 1220.

[13] C. K. Prier, D. A. Rankic, D. W. C. MacMillan, Chem.Rev. 2013, 113, 5322.

[14] D. A. Nicewicz, T. M. Nguyen, ACS Catal. 2014, 4, 355.

[15] J. Xie, H. Jina, P. Xu, C. Zhu, Tetrahedron Lett. 2014, 55,36 .
[16] X. Lang, X. Chen, J. Zhao, Chem. Soc. Rev. 2014, 43, 473.

[17] J. Hu, J. Wang, T. H. Nguyen, N. Zheng, Beilstein J. Org. Chem. 2013, 9, 1977.

[18] D. Rovelli, M. Fagnoni, A. Albini, Chem. Soc. Rev. 2013, 42, 97.

[19] T. P. Yoon, M. A. Ischay, J. Du, Nat. Chem. 2010, 2, 527.

[20] X.-J. Yang, B. Chen, L.-Q. Zheng, L.-Z. Wu, C.-H. Tung, Green Chem. 2014, 16, 1082.

[21] Y. C. Teo, Y. Pan, C. H. Tan, Chem. Cat. Chem. 2013, 5, 235.

[22] K. Fidaly, C. Ceballos, A. Falguières, M. S.-I. Veitia, A.Guy, C. Ferroud, Green Chem. 2012, 14, 1293.

[23] D.-T. Yang, Q.-Y. Meng, J.-J. Zhong, M. Xiang, Q. Liu, L.-Z. Wu, Eur. J. Org. Chem. 2013, 7528.

[24] Y.-Q. Zou, J.-R. Chen, X.-P. Liu, L.-Q. Lu, R. L. Davis, K. A. Jørgense, W.-J. Xiao, Angew. Chem. Int. Ed. 2012, 51,784 .

[25] D. P. Hari, B. König, Org. Lett. 2011, 13, 3852.

[26] M. Neumann, S. Füldner, B. König, K. Zeitler, Angew. Chem. Int. Ed. 2011, 50, 951.

[27] V. Rey, S. M. Soria-Catro, J. E. Arguello, A. B. Peñéñory, Tetrahedron Lett. 2009, 50, 4720.

[28] S. Gazi, R. Ananthakrishnan, RSC Adv. 2012, 2, 7781.

[29] J. Zhang, L. Wang, Q. Liu, Z. Yang, Y. Huang, Chem. Commun. 2013, 49, 11662.

[30] V. Srivastava, P. K. Singh, P. P. Singh, Chem. Heterocyclic Comp. 2014, 50, 573.

[31] V. Srivastava, P. K. Singh, P. P. Singh, Croat. Chem. Acta 2014, 87, 91.

[32] V. Srivastava, P. K. Singh, P. P. Singh, Croat. Chem. Acta 2015, 88, 59.

[33] V. Srivastava, P. K. Singh, P. P. Singh, Croat. Chem.Acta 2015, 88, 227.

[34] V. Srivastava, P. K. Singh, P. P. Singh, Asian Journal of Chemistry 2016, 28(10), 2159.

[35] V. Srivastava, P. K. Singh, Surabhi Sinha, P. P. Singh, Rev. Roum. Chim. 2016, 61(10), 755.

[36] Y. F. Liang, S. Song, L. Ai, X. Li, N. Jiao, Green Chem. 2016, 18, 6462.

[37] K. H. Popat, K. S. Nimavat, H. S. Vasoya, H. S. Joshi, Indian J. Chem. 2003, 42B, 1497.

[38] J. S. Ghomi, Z. Alishahi, J. Fudan Univ, Nat Sci, 2005, 44(5), 789.

[39] M. Yamaguchi, N. Maruyama, T. Koga, K. Kamei, M. Akima, T. Kuroki, M. Hamana, N. Ohi, Chem. Pharm. Bull, 1995, 43(2), 332.

[40] B. C. Kim, J. L. Kim, Y. U. Jhang, Bull Korean Chem Soc. 1994, 15(2), 97.

[41] T. Keshari, V. K. Yadav, V. P. Srivastava, L. D. S. Yadav, Green Chem. 2014, 16, 3986.

[42] F. R. Bisogno, A. Cuetos, I. Lavandera, V. Gotor, Green Chem. 2009, 11, 452.

[43] W. Fan, Q. Yang, F. Xu, P. Li, J. Org. Chem. 2014, 79. 
[44] D. C. Neckers, O. M. Valdes-Aguilera, Adv. Photochem. 1993, 18, 315.

[45] M. V. Encinas, A. M. Rufs, S. G. Bertolotti, C. M. Previtali, Polymer 2009, 50, 2762.

[46] T. Lizarides, T. McCormick, P. Du, G. Luo, B. Lindley, R. Eisenberg, J. Am. Chem. Soc. 2009, 131, 9192.
[47] S. H. Lee, D. H. Nam, C. B. Park, Adv. Synth. Catal. 2009, 351, 2589.

[48] T. Xiao, X. Dong, Y. Tang, L. Zhou, Adv. Synth. Catal. 2012, 354, 3195.

[49] G. K. Fekarurhobo, S. S. Angaye, F. G. Obomann, J. Emerg.Trends Engg. Appl. Sci. (JETEAS) 2013, 4, 394 